\title{
A UTILIZAÇÃo DE MATERIAIS OBTIDOS PELO PROCESSO DE SOL-GEL NA CONSTRUÇÃo DE BIOSSENSORES
}

\author{
Antonio A. S. Alfaya e Lauro T. Kubota*
}

Instituto de Química, Universidade Estadual de Campinas, CP 6154, 13083-970 Campinas - SP

Recebido em 17/8/01; aceito em 31/10/01

\begin{abstract}
THE UTILIZATION OF MATERIALS OBTAINED BY THE SOL-GEL PROCESS IN BIOSENSORS CONSTRUCTION. The use of sol-gel materials to develop new biosensors has received great attention due to its characteristics and versatility of sol-gel process. An overview is presented of the state-of-the-art of electrochemical biosensors employing sol-gel materials. Lowtemperature, porous sol-gel ceramics represent a new class for the immobilization of biomolecules. The rational design of solgel sensing materials, based on the judicious choice of the starting alkoxide, encapsulated reagents, and preparation conditions, allows tailoring of material properties in a wide range, and offers great potential for the development of electrochemical biosensors.
\end{abstract}

Keywords: biosensors; sol-gel process; electrochemical sensors.

\section{INTRODUÇÃO}

Atualmente muitos procedimentos analíticos necessitam de uma instrumentação complexa e pessoal altamente especializado. Os sensores químicos são dispositivos pequenos, robustos, portáteis, de fácil manipulação e não necessitam da adição contínua de reagentes para a sua operação podendo, assim, fornecer informações confiáveis continuamente. Portanto, o sensor químico tem sido um elemento chave na instrumentação analítica dispensando, em muitos casos, a utilização de aparelhos complexos e a necessidade de uma enorme infra-estrutura de suporte.

Os sensores eletroquímicos têm apresentado maior desenvolvimento nos últimos anos, devido à crescente necessidade de testes analíticos simples, rápidos e baratos para a determinação de importantes compostos químicos e biológicos em concentrações muito baixas. Os principais sensores eletroquímicos podem ser classificados como: potenciométricos, amperométricos, voltamétricos e condutométricos, sendo os sensores amperométricos os de maior destaque ${ }^{1-7}$.

Devido à crescente necessidade de identificação e quantificação de metabólitos de forma rápida, específica e em quantidades de amostras muito pequenas (líquidos corpóreos) no campo da medicina, ocorreu o desenvolvimento dos biossensores.

\section{OS BIOSSENSORES}

Os biossensores podem ser definidos como um sensor químico cujo reconhecedor é um componente biológico ativo, o que significa que um processo bioquímico é a fonte do sinal analítico. Assim uma das características dos biossensores, é a sua alta seletividade com relação a um determinado analito ${ }^{8,9}$.

A construção de um biossensor baseia-se na comunicação de duas partes: o componente biológico ativo (o reconhecedor) e um transdutor. A primeira faz o reconhecimento de uma determinada substância por meio de uma reação bioquímica específica, gerando uma variação na concentração de prótons, a liberação de gases, a emissão ou a absorção de luz, a emissão de calor, uma variação de

\footnotetext{
*e-mail: kubota@iqm.unicamp.br
}

massa, uma mudança no estado de oxidação do analito, etc; e a segunda, converte a energia destes eventos numa forma mensurável, como uma variação de corrente, potencial, temperatura e outros ${ }^{10}$. Assim, diferentes componentes biológicos podem ser utilizados na construção de biossensores, tais como organismos, tecidos, células, organelas, membranas, enzimas, receptores, anticorpos, ácidos nucléicos e macromoléculas orgânicas ${ }^{11-15}$.

Apesar de suas limitações em termos da sua susceptibilidade ao $\mathrm{pH}$, à força iônica, a inibidores e sua baixa estabilidade, as enzimas são amplamente utilizadas na construção de biossensores eletroquímicos ${ }^{16-20}$.

Vários métodos são utilizados na imobilização da enzima no material suporte para a construção de um biossensor. Entre eles, os mais utilizados são aqueles envolvendo adsorção, ligação covalente, ligação covalente cruzada com um reagente multifuncional e a oclusão em géis ou membranas ${ }^{21-25}$.

O método de adsorção para a imobilização de enzimas sobre materiais inorgânicos apresenta a grande vantagem da simplicidade de execução; no entanto, este processo apresenta grandes dificuldades, tais como:

(1) a reprodução da forma da enzima adsorvida sobre o material utilizado, pois a enzima só pode manter a sua atividade se os seus centros ativos encontrarem-se em uma forma disponível sobre a superfície do material, para que a reação com o analito específico possa ocorrer;

(2) a lixiviação da enzima durante o processo de medida, uma vez que a enzima se fixa ao substrato, geralmente por ligações fracas passíveis de serem rompidas durante o uso do biossensor pela interação com o solvente e/ou a variação de $\mathrm{pH}$ e temperatura;

(3) o "envenenamento" rápido do eletrodo, devido ao fato da enzima se encontrar bastante exposta ao meio a ser analisado;

(4) a baixa estabilidade da enzima, devido aos fatores supramencionados e também deformação da estrutura tridimensional da enzima durante o tempo de uso do biossensor.

O método da ligação covalente consiste na formação de ligações entre o substrato e a enzima. Estas ligações são geralmente feitas pela reação de grupos funcionais específicos da enzima, os quais não são essenciais para a sua atividade catalítica com grupos reativos específicos presentes no suporte sendo, portanto, necessário um conhecimento prévio da estrutura química da enzima e da natureza do 
suporte. As grandes vantagens deste método são a minimização da lixiviação e uma maior estabilidade do complexo enzima-suporte em relação aos efeitos da variação do $\mathrm{pH}$, da força iônica, do solvente. Entretanto, o método apresenta alta possibilidade de perda da atividade enzimática devido à reação com grupos funcionais do centro ativo da enzima, como também pela deformação da estrutura tridimensional da mesma.

O terceiro processo é o mais amplamente utilizado e baseia-se na formação de ligações cruzadas entre os grupos amino do suporte com os grupos amino da enzima, ou da formação de ligações cruzadas intermoleculares com a formação de partículas insolúveis macroscópicas, pela utilização de reagentes bi- ou multifuncionais. As principais vantagens deste método são a simplicidade de execução e a forte interação da enzima com o suporte, diminuindo drasticamente a lixiviação e proporcionando ao eletrodo uma maior estabilidade. Entretanto, também apresenta grandes desvantagens, como a dificuldade do controle da reação, o uso de grandes quantidades de enzima, baixa atividade enzimática e dificuldades na reprodutibilidade do eletrodo, levando assim a uma baixa reprodutibilidade no desempenho do eletrodo.

O procedimento de imobilização por oclusão em géis ou membranas orgânicas corresponde ao confinamento do material biológico em uma matriz polimérica ou em uma membrana semipermeável, sendo o sistema revestido por uma membrana polimérica permeável, para reduzir a lixiviação do material biológico. Este tipo de imobilização apresenta a grande vantagem de se poder utilizar qualquer tipo de enzima. No entanto, exibe a desvantagem de lixiviação do material enzimático, devido aos diferentes tamanhos de poros nos polímeros, como também problemas de tempo de resposta, pela dificuldade de difusão das espécies envolvidas através das membranas.

\section{O PROCESSO DE SOL-GEL}

O uso do processo de sol-gel para produzir materiais sensores para aplicações analíticas tem atraído considerável interesse dos pesquisadores ${ }^{26}$. Isto é devido a numerosos fatores, como a facilidade de fabricação, a flexibilidade do projeto de síntese e o fato das enzimas ocluídas na matriz de sol-gel manterem a sua respectiva atividade catalítica $^{27-31}$.

O processo de sol-gel pode ser facilmente reconhecido, pois trata de qualquer rota de síntese de materiais onde num determinado momento ocorre uma transição do sistema sol para um sistema gel. O termo sol é empregado para definir uma dispersão de partículas coloidais (dimensão entre 1 e $100 \mathrm{~nm}$ ) estável em um fluido, enquanto o termo gel pode ser visto como sendo um sistema formado pela estrutura rígida de partículas coloidais (gel coloidal) ou de cadeias poliméricas (gel polimérico) que imobiliza a fase líquida nos seus interstícios. Desse modo, os géis coloidais resultam da agregação linear de partículas primárias (Figura 1a), que só pode ocorrer pela alteração apropriada das condições físico-químicas da suspensão. Por outro lado, os géis poliméricos são, geralmente, preparados a partir de soluções onde se promovem reações de polimerização. Neste caso a gelatinização ocorre pela interação entre as longas cadeias poliméricas lineares (Figura 1b) ${ }^{27}$.

O processo de sol-gel pode ser dividido em duas classes, dependendo da natureza do precursor inorgânico utilizado: a dos sais (cloretos, nitratos, sulfetos, etc.) e a dos alcóxidos. A rota que envolve o uso de precursores do tipo alcóxido aparece como a mais versátil atualmente. A hidrólise de uma solução de tetraalcoxissilanos em um solvente orgânico, como o álcool, leva à formação de partículas com função silanol, as quais formam um sol pela polimerização via condensação, e a continuação do processo leva a um gel. Esta transformação é designada transição sol-gel. Após secagem do gel, um

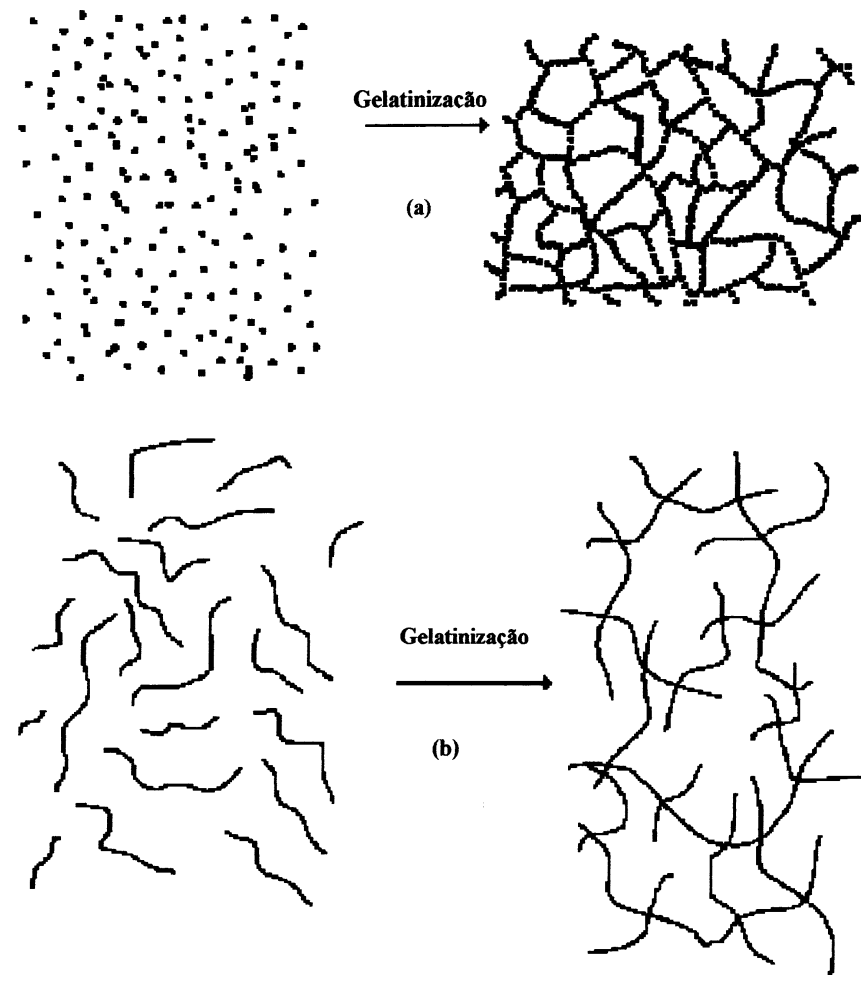

Figura 1. Esquema da transição sol-gel: (a) formação de gel particulado e (b) formação de gel polimérico

xerogel é formado. As reações químicas que ocorrem durante a formação do sol, do gel e do xerogel influenciam fortemente a composição e as propriedades do produto final ${ }^{28-30}$. Uma compreensão básica da cinética e das reações envolvidas no processo de sol-gel é de primordial importância na preparação de materiais com características controladas.

A reação de polimerização sol-gel pode ser dividida em duas etapas básicas:

(1) a hidrólise do grupo alcóxido com a formação de grupos reativos do tipo silanol

$\mathrm{Si}(\mathrm{OR})_{4}+n \mathrm{H}_{2} \mathrm{O} \longrightarrow \mathrm{Si}(\mathrm{OR})_{4-n}(\mathrm{OH})_{n}+n \mathrm{ROH}$,

(2) a condensação do grupo silanol, a qual leva inicialmente à formação do sol e, eventualmente, ao gel $^{28-30}$

$\equiv \mathrm{Si}-\mathrm{OH}+\mathrm{HO}-\mathrm{Si} \equiv \longrightarrow \equiv \mathrm{Si}-\mathrm{O}-\mathrm{Si} \equiv+\mathrm{H}_{2} \mathrm{O}$

ou

$\equiv \mathrm{Si}-\mathrm{OR}+\mathrm{HO}-\mathrm{Si} \equiv \longrightarrow \mathrm{Si}-\mathrm{O}-\mathrm{Si} \equiv+\mathrm{ROH}$.

Do mecanismo de sol-gel apenas a primeira etapa, a hidrólise, é bem conhecida, pois as reações de condensação começam antes das reações de hidrólise terminarem, tornando o mecanismo muito complexo e envolvendo muitas reações de hidrólise e condensação ao mesmo tempo ${ }^{28-31}$.

As reações de hidrólise e condensação ocorrem via substituição nucleofílica bimolecular no átomo de silício $\left(\mathrm{S}_{\mathrm{N}} 2-\mathrm{Si}\right)$. Como os alcóxidos de silício possuem uma baixa reatividade (relativa aos alcóxidos metálicos), catalisadores ácidos $\left(\mathrm{H}_{3} \mathrm{O}^{+}\right)$, básicos $\left(\mathrm{OH}^{-}\right)$e/ou nucleofílicos ( $\mathrm{F}^{-}, \mathrm{N}$-metilimidazol, hexametilfosforamida - HMPA) 
são adicionados ao processo para promover um aumento na velocidade das reações de hidrólise e condensação ${ }^{27-30}$. A reação de hidrólise sob condições ácidas envolve a protonação do grupo alcóxido, seguida pelo ataque nucleofílico da água, para formar um intermediário penta-coordenado. A carga positiva sobre o grupo alcóxido confere a ele um caráter de um grupo de saída melhor ${ }^{28-31}$. Sob condições básicas, acredita-se que o mecanismo envolva o ataque nucleofílico sobre o átomo de silício pelo ânion hidróxido para formar um intermediário penta-coordenado carregado negativamente, seguido pela saída de um ânion alcóxido ${ }^{28-31}$.

As propriedades físicas (isto é, área superficial específica, tamanho médio dos poros e sua distribuição) de um gel seco (o xerogel) dependem fortemente dos parâmetros utilizados no processo de solgel. A razão molar água:silano, a natureza e a concentração do catalisador e o tipo do precursor alcóxido são parâmetros específicos que afetam fortemente as velocidades relativas das reações de hidrólise e condensação, as quais, por sua vez, ditam as propriedades do material final. Em geral, preparações com baixo valor de $\mathrm{pH}$ e baixo teor de água produzem materiais densos com tamanho médio de poros pequeno, enquanto preparações com valores altos de $\mathrm{pH}$ e altos teores de água produzem materiais mais porosos ${ }^{28-30,32}$. Sob condições de catálise ácida, a condensação ocorre preferencialmente entre os grupos silanóis localizados em monômeros ou no final de cadeias poliméricas. Isto leva à formação de géis compostos de cadeias poliméricas lineares entrelaçadas, as quais, após secagem formam uma matriz de baixo volume de poro. Sob condições básicas, a condensação ocorre preferencialmente entre oligômeros altamente ramificados, os quais, levam à formação de géis particulados que, após secagem, produzem materiais com alta porosidade ${ }^{28,29,32}$. A velocidade das reações de hidrólise e condensação é muito influenciada também pelo tamanho do grupo alcóxido, devido a fatores estéricos. O aumento no tamanho do grupo alcóxido e o maior número de ramificações diminuem a velocidade de hidrólise ${ }^{28-30}$.

Existe uma certa dificuldade de se combinar compostos orgânicos e inorgânicos, a qual se baseia no fato de que os componentes orgânicos não são estáveis nas altas temperaturas em que normalmente se trabalha com os componentes inorgânicos. Para contornar este problema os pesquisadores têm utilizado o processo de sol-gel e obtido ótimos resultados. Este processo, que tem como característica o processamento a baixas temperaturas, oferece a possibilidade de obtenção de materiais híbridos, uma vez que nestas temperaturas os componentes orgânicos não sofrem decomposição $0^{33}$.

As maneiras de incorporar materiais orgânicos em matrizes inorgânicas provenientes do processo de sol-gel podem ser convenientemente divididas em três grupos ${ }^{34}$. No primeiro grupo, a morfologia contínua do poro da matriz inorgânica é explorada para a impregnação de materiais orgânicos e, no segundo grupo, os componentes orgânicos são dispersos no sol e após a gelatinização esses materiais orgânicos são presos dentro da matriz do óxido. Nestes dois métodos não existe a formação de ligações covalentes entre os óxidos e os componentes orgânicos. No terceiro grupo, um componente orgânico está ligado covalentemente $\left(\mathrm{R}_{4-\mathrm{x}} \mathrm{Si}(\mathrm{OR})_{\mathrm{x}}\right.$, onde $\left.\mathrm{x}=1-4\right)$ ao precursor inorgânico, o qual formará uma rede rígida com o componente orgânico ligado quimicamente na estrutura inorgânica, tornandose parte de uma rede integrada; estes materiais são chamados de ORMOSIL ("Organically Modified Silicates"). Um ormosil é, portanto, um material híbrido inorgânico/orgânico em escala molecular, sendo geralmente um nanocompósito. Esses mesmos materiais têm sido chamados também de "ormocers" ou de "ceramers" $34-36$.

A complexidade associada com o processo de polimerização torna-se ainda mais clara quando da preparação de materiais a partir de precursores organoalcoxissilanos ${ }^{31,33}$. Aqui, as velocidades relativas de hidrólise e condensação são fortemente dependentes de fatores estéricos e indutivos ${ }^{30,31,37}$. Devido aos mecanismos de reação sob condições ácidas e básicas envolverem a formação de estados de transição carregados positiva ou negativamente, respectivamente, o efeito do substituinte alquila sobre as velocidades de hidrólise deverá ser visivelmente afetado pela natureza do catalisador ${ }^{28-30,37}$. Por exemplo, a substituição de um ou mais grupos metila indutores de elétrons por grupos alcóxidos aumenta a densidade eletrônica sobre o silício. Sob condições ácidas, este fato proporciona o aumento da velocidade de hidrólise devido à estabilização do estado de transição que está carregado positivamente ${ }^{28-30,37}$. No entanto, sob condições básicas, uma significativa diminuição na velocidade é observada, devido à desestabilização do estado de transição carregado negativamente. Assim, a reatividade química individual do precursor organoalcoxissilano é de suma importância na formação do material híbrido orgânico/ inorgânico. Se o grupo funcional orgânico possui propriedades ácidas ou básicas ele vai influenciar o processo de hidrólise e condensação, tornando a polimerização ainda mais complexa ${ }^{38}$.

Precursores de fórmula geral, $\mathrm{R}_{4-\mathrm{x}} \mathrm{Si}(\mathrm{OR})_{\mathrm{x}}$, onde $\mathrm{x}=1-4$, são obtidos comercialmente ou podem ser preparados via diferentes rotas sintéticas $^{31,38,39}$. O grupo funcional R está ligado ao átomo de Si por uma ligação Si-C, a qual é bastante resistente à hidrólise devido à baixa acidez de Lewis do silício. Os trialcoxissilanos $(x=3)$ são utilizados como modificadores de rede para melhorar propriedades específicas, como a hidrofobicidade, a reatividade e a flexibilidade na rede inorgânica. Estes precursores podem ser polimerizados sozinhos ou preferencialmente com tetraalcoxissilanos $(\mathrm{x}=4)$, os quais melhoram a conectividade da rede e a estabilidade do sólido final. Dialcoxissilanos $(\mathrm{x}=2)$ formam espécies cíclicas ou cadeias nas condições de hidrólise e condensação não possibilitando a formação de ligações cruzadas na rede. No entanto, quando se polimerizam estes precursores na presença de tetra ou trialcoxissilanos, eles atuam como modificadores de rede e promovem a flexibilidade na matriz ${ }^{30,38}$. Embora se possa exercer o controle das reações de hidrólise e condensação de forma a promover uma distribuição homogênea do modificador de rede na matriz, isto não é sempre o que ocorre. Por exemplo, quando trialcoxissilanos são polimerizados sem a adição de qualquer outro formador de rede, acredita-se que o grupo orgânico se localize na superfície e não homogeneamente pela matriz ${ }^{30}$. Sob condições básicas, a velocidade de hidrólise para os organoalcoxissilanos pode ser de várias ordens de magnitude menor que para os tetraalcoxissilanos. Assim, pode-se promover a formação de materiais nos quais os grupos orgânicos estão localizados na superfície da matriz. A formação de materiais heterogêneos, particularmente na escala nanoscópica, não é necessariamente um fato desfavorável, se o fenômeno puder ser controlado. Entretanto, isto deverá exigir um conhecimento detalhado das velocidades das reações dos precursores sob o conjunto de condições específicas utilizadas na rota de sol-gel.

A estratégia mais comum nos dias de hoje na preparação de materiais sensores pelo processo de sol-gel tem envolvido, em primeiro lugar, uma boa idéia inicial do objetivo a ser alcançado e, em segundo, um processo laborioso combinando tentativas e erros com uma boa intuição química.

\section{BIOSSENSORES ELETROQUÍMICOS EMPREGANDO O PROCESSO DE SOL-GEL}

Braun et al. ${ }^{40,41}$ ocluíram diversas enzimas em silicatos produzidos pelo processo de sol-gel, pela mistura das macromoléculas com os precursores adequados em meio reacional com baixo teor de metanol. Surpreendentemente, as enzimas resistiram às condições de polimerização inorgânica, não sendo totalmente desnaturadas pelo solvente alcoólico empregado e permanecendo ativas, mesmo após a contração da estrutura porosa durante a etapa de secagem do mate- 
rial. Ellerby et al..$^{42}$ foram os primeiros a utilizar o ultra-som na solução de alcoxissilano com o objetivo de preparar o silicato na fase sol, o qual continha muito pouco ou nenhum álcool. As enzimas foram então misturadas com o sol em um processo de sol-gel convencional. Em muitos casos as enzimas ocluídas foram muito mais estáveis do que na forma solúvel, presumivelmente pela formação de pontes de hidrogênio com os grupos silanóis, os quais inibem a reconformação térmica ${ }^{43}$. Uma notável realização foi efetuada por Reetz e colaboradores ${ }^{44}$, pela oclusão de lipases em silicatos hidrofobicamente modificados. As lipases são amplamente utilizadas em reações de esterificação orgânica em meio hidrofóbico. As lipases ocluídas pelo processo de sol-gel apresentam alta estabilidade e uma reatividade comparável com as enzimas isoladas devido à interação hidrofóbica com o suporte de ormosil. Atualmente, lipases ocluídas pelo processo de sol-gel podem ser adquiridas comercialmente.

Mais de 35 tipos diferentes de materiais híbridos cerâmicosbioquímicos foram tabelados, em 1994, numa publicação sobre biocerâmicas produzidas pelo processo de sol-gel ${ }^{45}$. Enzimas, anticorpos, células inteiras e extratos de células e outros materiais biológicos ativos foram ocluídos em uma variedade muito grande de matrizes inorgânicas incluindo sílica ${ }^{46-49}$, zircônia ${ }^{50,51}$, titânia ${ }^{51}$, alumina $^{52} \mathrm{e}$ compósitos de celulose-titânia ${ }^{49,53}$, sílica-grafite ${ }^{49,54-56}$ e sílica-ouro ${ }^{57}$.

Muitos estudos eletroquímicos se concentraram nos biossensores para glicose, devido à alta estabilidade da glicose oxidase durante o processo de sol-gel e pela importância no desenvolvimento de um sensor para a glicose no campo das pesquisas médicas ${ }^{58}$. Biossensores eletroquímicos baseados na glicose oxidase funcionam conforme o seguinte mecanismo: a D-glicose é oxidada para a gluconolactona pela redução de um grupo flavina. A flavina reduzida pode então ser oxidada novamente pelo oxigênio, o qual é convertido em peróxido de hidrogênio. $\mathrm{O}$ consumo do oxigênio ou a oxidação anódica do peróxido de hidrogênio pode ser utilizado para a quantificação da glicose. A etapa de regeneração da enzima é promovida por um par redox (um mediador de elétrons, na maioria das vezes), o qual pode ser eletroquimicamente regenerado por uma etapa de oxidação sobre a superfície do eletrodo. Pela utilização deste método, o sinal não é fortemente dependente da concentração de oxigênio, e como é possível ocorrer um processo de detecção e regeneração a baixas voltagens, então a interferência de outras espécies químicas é bastante minimizada.

Tatsu et al ${ }^{59}$ produziram uma sílica contendo glicose oxidase e uniram-na a um eletrodo de oxigênio por meio de uma membrana de nylon. O decréscimo da concentração de oxigênio foi então utilizado para quantificar o teor de glicose. Audebert et al. ${ }^{60}$ desenvolveram um biossensor contendo a glicose oxidase e o ferroceno (como mediador) incorporados a um filme fino de sílica, produzido pelo processo de sol-gel, e este foi depositado sobre um suporte metálico inerte. Entretanto, somente $80 \%$ da atividade total da enzima foi mantida no gel. Essa técnica está limitada a géis úmidos pois, quando o xerogel é formado, a enzima perde a sua atividade.

Narang et $a l^{61}$ imobilizaram a enzima glicose oxidase entre duas camadas de filmes finos de sílica, produzidos pelo processo de solgel, numa construção tipo sanduíche depositado sobre um substrato condutor de ITO (ITRIUM TIN OXIDE), sendo o peróxido de hidrogênio produzido detectado eletroquimicamente. Entretanto, o sinal deste tipo de eletrodo é pequeno, na maioria dos casos. Li et al. ${ }^{62}$ imobilizaram a peroxidase de raiz forte em um eletrodo de pasta de carbono e depositaram uma camada de ormosil sobre o eletrodo; Yang et al. ${ }^{63}$ prepararam um biossensor em miniatura recobrindo um fio de platina com glicose oxidase imobilizada sobre micro-esferas preparadas por sol-gel.

A imobilização de enzimas no interior de matrizes produzidas pelo processo de sol-gel é uma técnica muito vantajosa, pois preser- va a atividade enzimática e evita a lixiviação da enzima e de moléculas pequenas dos mediadores de elétrons para a solução, ao contrário do que ocorre, na maioria das vezes, com filmes finos ou membranas contendo estes mesmos materiais imobilizados. Park et al. ${ }^{64}$ desenvolveram um novo processo de sol-gel para prevenir a lixiviação das espécies de interesse como a lactato oxidase e a glicose oxidase e polímeros redox $\left[(\mathrm{Os})(\mathrm{bpi})_{2}(\mathrm{PVP})_{10} \mathrm{Cl}\right] \mathrm{Cl}$, partindo de poli(4vinilpiridina) em um filme fino de ormosil produzido por sol-gel.

Muitos pesquisadores têm se dedicado a pesquisar materiais suportes para biossensores de natureza diferente da sílica e produzidos pelo processo de sol-gel. Glezer e Lev ${ }^{49,65}$ desenvolveram um método para a oclusão da glicose oxidase e da peroxidase de raiz forte em um gel de pentóxido de vanádio. Os filmes finos de $\mathrm{V}_{2} \mathrm{O}_{5}$ apresentam boa condutividade elétrica, além de boas propriedades de aderência a superfícies condutoras como o carbono vítreo e a platina. Liu et al. $^{52}$ desenvolveram um novo biossensor co-imobilizando o $\mathrm{Fe}(\mathrm{CN})_{6}{ }^{4-}$ como mediador de elétrons e a tirosinase em uma matriz de $\mathrm{Al}_{2} \mathrm{O}_{3}$ porosa, produzida pelo processo de sol-gel.

A construção de biossensores com a utilização de compósitos carbono-cerâmicos é uma variação no processo de sol-gel, a qual visa uma maior proteção para a enzima durante a síntese do sol-gel e também contribui para a diminuição da lixiviação das moléculas de mediadores de elétrons (moléculas pequenas) e das próprias enzimas para a solução. Os biossensores construídos com compósitos carbono-cerâmicos apresentam uma lenta lixiviação dos mediadores somente após longos períodos de utilização ${ }^{66,67}$.

Existem três variações principais utilizadas pelos pesquisadores para a construção de biossensores tipo compósito carbono-cerâmico, com o objetivo de se minimizar ao máximo a lixiviação dos mediadores de elétrons.

(1) Oclusão da enzima quimicamente modificada com o mediador no compósito carbono-cerâmico ${ }^{68}$. Nesta construção, muitos grupos ferroceno estão ligados covalentemente na enzima glicose oxidase e, então, a enzima é ocluída na matriz carbono-cerâmica produzida pelo processo de sol-gel. A lixiviação das moléculas de mediador é eliminada por elas se encontrarem ligadas covalentemente à estrutura da enzima e imobilizada no interior da rede inorgânica.

(2) Oclusão de oxidoredutases em compósito carbono-cerâmico quimicamente modificado com mediadores ${ }^{69,70}$. O material do eletrodo é constituído de uma dispersão de grafite em pó e glicose oxidase incorporada em um silicato estrutural multifuncional modificado com ferroceno, contendo grupos amina e metil, preparado pela copolimerização de vários precursores em um processo de sol-gel (Figura 2). Cada espécie nesta construção integrada realiza uma tarefa específica. A

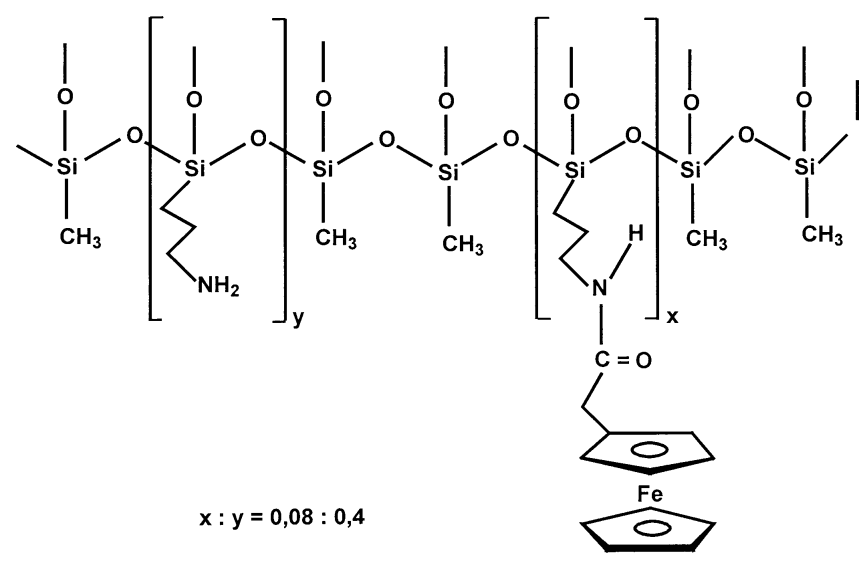

Figura 2. Estrutura de um silicato modificado com ferroceno 
grafite melhora a condutividade por percolação; o silicato fornece uma estrutura rígida altamente reticulada, a qual aprisiona a enzima redox; os grupos ferroceno são responsáveis pelo transporte do sinal dos centros ativos da enzima até a superfície condutora; os grupos amina foram incorporados devido à sua alta afinidade ao excesso de cargas negativas sobre a superfície da glicose oxidase e, finalmente, a combinação dos grupos metil e amina é vantajosa para o controle da hidrofilicidade da secção eletroativa do eletrodo.

(3) Imobilização da oxidoredutase em um compósito de grafite modificado com mediador-silicato ${ }^{71}$. Este método está baseado na ligação covalente do mediador de elétrons sobre a grafite em pó. A grafite foi primeiramente recoberta com ouro e, a seguir, uma reação de derivatização foi realizada (Figura 3). Estudos ciclovoltamétricos demonstram sensibilidade na resposta, mesmo para espaçadores longos.

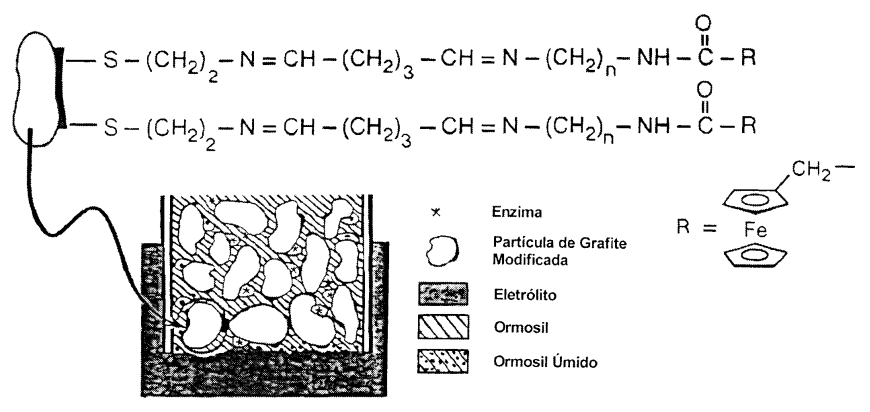

Figura 3. Estrutura da partícula de grafite modificada com ferroceno e o esquema de um biossensor tipo compósito carbono-cerâmico

A interferência química devido ao sobrepotencial aplicado ao biossensor construído a partir de um compósito carbono-cerâmico é um dos problemas mais freqüentes quando se trabalha com estes materiais. Este inconveniente pode ser bastante minimizado com a utilização de catalisadores metálicos no compósito. Os metais mais utilizados com esta finalidade são o paládio ${ }^{72}$, o ródio ${ }^{73}$ e o rutênio ${ }^{74}$, sendo o último geralmente utilizado na fabricação de eletrodos constituídos de um filme fino, produzido pelo processo de sol-gel. Em todos estes $\operatorname{casos}^{72-74}$, o tempo de meia-vida foi superior a 4 meses. Os óxidos de rutênio ${ }^{74}$ e de ródio ${ }^{73}$ apresentam extraordinária seletividade para a glicose quando na presença de compostos bioquímicos comuns, como o ácido ascórbico, acetaminofeno e ácido úrico. Biossensores para lactato (à base de lactato oxidase) e amino ácidos (à base de amino ácido oxidase) foram preparados de maneira semelhante $^{72}$. Um fato muito interessante e de ordem prática, dos eletrodos compósito carbono-cerâmico é que a secção ativa do eletrodo não é destruída por sucessivos polimentos devido à dureza da estrutura do silicato formado pelo sol-gel e assim, a secção ativa do eletrodo pode ser renovada por polimento mecânico após o uso constante ou a contaminação deste.

Wang et al. $^{75}$ demonstraram que tiras teste para detecção de peróxido de hidrogênio e glicose podem ser produzidas pelo processo de sol-gel utilizando a técnica "screen-printing".

A utilização do processo de sol-gel para a fabricação de biossensores nos últimos anos tem revelado a grande flexibilidade desta técnica. Os pesquisadores estão investigando não só as diversas formas de imobilização das enzimas em diversos materiais inorgânicos, como também, uma grande atenção está sendo dirigida para os mais diversos tipos de transdutores. A Tabela 1 mostra alguns exemplos de biossensores obtidos pelo processo de sol-gel.

Um fato interessante sobre a versatilidade do processo de sol-gel pode ser demonstrado nos últimos avanços tecnológicos para a fabricação de biossensores inteligentes. Os pesquisadores estão se dedicando à pesquisa de biossensores com materiais biocompatíveis produzidos pelo processo de sol-gel, os quais são capazes de quantificar um determinado metabólito na corrente sangüínea e com um "chip" acoplado são capazes de analisar este dado e regular a concentração

Tabela 1. Exemplos de biossensores utilizando materiais sol-gel

\begin{tabular}{|c|c|c|c|c|c|}
\hline Analito & Tipo do biossensor & Enzima & Descrição & Limite de detecção & Ref. \\
\hline $\mathrm{H}_{2} \mathrm{O}_{2}$ & óptico & HRP & Membrana de silicato & $8 \times 10^{-6} \mathrm{~mol} \mathrm{~L}^{-1}$ & 85 \\
\hline Glicose & amperométrico & GOx & Microeletrodo & $500 \mathrm{ppm}$ & 86 \\
\hline Fenol & óptico & HRP & Membrana de silicato & - & 87 \\
\hline $\mathrm{H}_{2} \mathrm{O}_{2}$ & amperométrico & HRP & Polímero redox de ósmio & $5 \times 10^{-6} \mathrm{~mol} \mathrm{~L}^{-1}$ & 88 \\
\hline $\mathrm{NO}_{2}^{2}$ & óptico & Domínio heme da SGC & Fibra óptica recoberta & $111 \times 10^{-6} \mathrm{~mol} \mathrm{~L}^{-1}$ & 89 \\
\hline Uréia & condutométrico & Urease & "screen-printed" & $30 \times 10^{-6} \mathrm{~mol} \mathrm{~L}^{-1}$ & 90 \\
\hline Fenol & amperométrico & TPH & Medidas a $40^{\circ} \mathrm{C}$ & $2,5 \times 10^{-6} \mathrm{~mol} \mathrm{~L}^{-1}$ & 91 \\
\hline E. coli & amperométrico & Enterotoxina & Filme fino & $3,6 \times 10^{-8} \mathrm{~mol} \mathrm{~L}^{-1}$ & 92 \\
\hline $\begin{array}{c}\text { Acetiltiocolina e } \\
\text { Tiocolina }\end{array}$ & amperométrico & $\mathrm{ACHE} / \mathrm{CHO}$ & $\begin{array}{l}\text { Reator de vidro } \\
\text { modificado }\end{array}$ & $5 \times 10^{-6} \mathrm{~mol} \mathrm{~L}^{-1}$ & 93 \\
\hline Uréia & condutométrico & urease & Microeletrodo & $2 \times 10^{-4} \mathrm{~mol} \mathrm{~L}^{-1}$ & 94 \\
\hline Uréia & condutométrico & urease & Filme fino & $3 \times 10^{-6} \mathrm{~mol} \mathrm{~L}^{-1}$ & 90 \\
\hline $\begin{array}{l}\text { Organofosfatos/ } \\
\text { Acetilcolina }\end{array}$ & potenciométrico & ACHE/OPH & Microeletrodo & - & 95 \\
\hline $\mathrm{H}_{2} \mathrm{O}_{2}$ & amperométrico & HRP & Filme fino/FIA & $5 \times 10^{-7} \mathrm{~mol} \mathrm{~L}^{-1}$ & 96 \\
\hline $\mathrm{PH}^{2}$ & óptico & lipossomas & Filme fino & 0,01 unidade de $\mathrm{pH}$ & 97 \\
\hline $\mathrm{H}_{2} \mathrm{O}_{2}$ & amperométrico & HRP & Filme fino & $13,5 \times 10^{-6} \mathrm{~mol} \mathrm{~L}^{-1}$ & 98 \\
\hline $\mathrm{H}_{2} \mathrm{O}_{2}$ & amperométrico & HRP & Membrana & $9 \times 10^{-7} \mathrm{~mol} \mathrm{~L}^{-1}$ & 99 \\
\hline Álcoois e Aldeídos & fluorescência & $\mathrm{ADH}$ & Enzima ocluída & $\begin{array}{l}1 \times 10^{-4} \mathrm{~mol} \mathrm{~L}^{-1} \\
\text { (fase aquosa) } \\
1 \times 10^{-6} \mathrm{~mol} \mathrm{~L}{ }^{-1} \\
\text { (fase vapor) }\end{array}$ & 100 \\
\hline
\end{tabular}

As siglas utilizadas significam: HRP (peroxidase de raiz forte), GOx (glicose oxidase), SGC (guanilato ciclase solúvel), TPH (fenol hidrolase termoestável), ACHE (acetilcolinesterase), CHO (colina oxidase), OPH (organofosfato hidrolase) 
deste metabólito pela eliminação na corrente sangüínea de uma determinada substância. Um biossensor deste tipo está sendo desenvolvido por Gerritsen et al. ${ }^{76}$ que estudam a biocompatibilidade de sensores para a glicose, implantados de forma subcutânea e que foram recobertos por materiais inorgânicos produzidos pelo processo de sol-gel. Outro caminho que está sendo seguido pelos pesquisadores para o desenvolvimento de biossensores inteligentes é o desenvolvimento de técnicas de imobilização de materiais bi-lamelares lipídicos (membranas lipídicas) em matrizes produzidas pelo processo de sol-gel, com o objetivo de desenvolvimento de biossensores ópticos de alto desempenho para a análise e o controle de metabólitos importantes na corrente sangüínea ${ }^{77}$. $\mathrm{O}$ objetivo destes pesquisadores é desenvolver não apenas biossensores, mas sensores inteligentes que, além de determinar o analito, possam eliminar substâncias reguladoras na corrente sangüínea para o controle contínuo de certas doenças, como a diabetes.

\section{CONCLUSÕES}

A tecnologia do sol-gel está provando ser uma ferramenta altamente eficiente na produção de materiais com as mais diversas características físico-químicas, para as mais diversas aplicações analíticas e, principalmente, no desenvolvimento de biossensores. A palavra chave que melhor descreve a tecnologia de sol-gel é a versatilidade, pois oferece métodos simples de manipulação da textura, estrutura, configuração, composição e características químicas dos materiais produzidos. A facilidade de manipulação da forma final do material e a capacidade de imobilizar moléculas biológicas mantendo as suas características funcionais faz do processo de sol-gel uma tecnologia de futuro. Pois abre a possibilidade da miniaturização dos biossensores para medidas em sistemas in situ em organismos vivos, como também se torna uma tecnologia possível para o desenvolvimento de biossensores eletroquímicos multianalíticos.

Os materiais sensores produzidos até hoje têm servido praticamente como um suporte microporoso para a detecção de analitos específicos. Pequena atenção foi dedicada à construção racional dos materiais produzidos pelo processo de sol-gel, os quais podem ser manufaturados para se obter inúmeras propriedades específicas, tais como controle da polaridade superficial; ajuste da acidez do material para facilitar o processo de imobilização por troca iônica, aumentando a fixação de moléculas interessantes para a melhoria da atividade sensora; a utilização de precursores alcóxidos modificados ${ }^{78,79}$ com moléculas mediadoras ${ }^{70}$ para que a lixiviação do material seja eliminada e a utilização de precursores modificados com longas cadeias hidrofóbicas ${ }^{44,80}$ para possibilitar uma maior flexibilidade na rede inorgânica, proporcionando um controle maior sobre o tamanho dos poros do material, o que facilitaria a difusão do analito até o reconhecedor biológico, como também facilitaria que a integridade da estrutura tridimensional da enzima fosse mantida, preservandose ao máximo a sua atividade catalítica.

A polimerização sol-gel do tipo "template" (Figura 4) vem apresentando considerável interesse na última década. Os materiais produzidos por esta técnica apresentam uniformidade na forma e no tamanho dos poros. Isto é obtido graças a um processo de polimerização sol-gel ao redor de uma molécula ou uma estrutura inerte (um "template"), a qual pode ser removida após a formação do material, produzindo neste micro e meso cavidades regulares na forma e no tamanho. Desta forma, o processo produz após a remoção da molécula hospedeira, materiais micro ou mesoporosos uniformes ${ }^{81,82}$. A reação sol-gel do tipo "template" deverá ser estudada melhor para aumentar a sensibilidade dos biossensores eletroquímicos ${ }^{81,82}$, pois a construção de materiais com a forma e o tamanho de poros definidos irá criar uma classe de materiais sensores altamente interessantes, pois será possível a seleção das estruturas que poderão entrar em

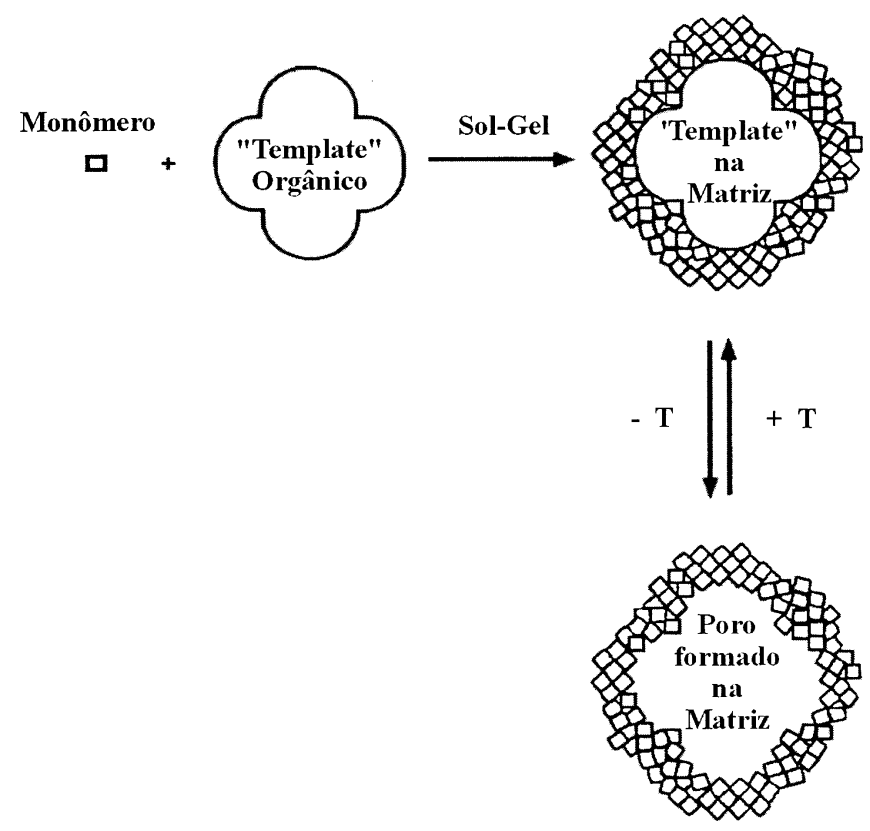

Figura 4. Esquema de polimerização sol-gel do tipo "template"

contato com o material biológico utilizado, criando barreiras de difusão para espécies não desejáveis e por conseguinte aumentando a sensibilidade e o tempo de utilização dos biossensores.

A formação de novos compósitos baseados no acoplamento de matrizes sol-gel e outros processos poderá ser muito benéfica para as futuras aplicações dos biossensores. Alguns exemplos destes acoplamentos de técnicas podem ser evidenciados pela fixação de matrizes produzidas por sol-gel sobre eletrodos de ouro através da utilização de precursores alcóxidos com a função tiol ${ }^{83}$; a eletropolimerização de filmes (1,2-diaminobenzeno $)^{84}$ sobre materiais porosos produzidos pelo processo de sol-gel pode criar novos compósitos para a construção de biossensores com elevada sensibilidade; a construção de biossensores com tempo de resposta extremamente curto e com alta sensibilidade pode ser realizada pela polimerização de polianilinas no interior de redes inorgânicas produzidas pelo processo de sol-gel ${ }^{85}$.

O estudo de materiais diferentes do silicato pelo processo de solgel e a sua aplicação na construção de biossensores é um campo muito amplo e de grandes possibilidades futuras de sucesso, entretanto poucos pesquisadores têm se aventurado, devido à pouca pesquisa fundamental existente neste campo.

Os recentes desenvolvimentos na área dos biossensores com utilização de materiais produzidos pelo processo de sol-gel são extremamente promissores, no entanto um grande esforço na pesquisa fundamental para o desenvolvimento de novos materiais sol-gel com características específicas deverá ser realizado pois, uma melhor compreensão da correlação entre o tipo de precursor utilizado, as condições de reação e as propriedades físico-químicas do material final são extremamente importantes na construção e no desempenho dos biossensores. No atual nível de interesse e desenvolvimento de materiais produzidos pelo processo de sol-gel para a construção de biossensores, pode-se observar o início de um amplo campo de pesquisa, que vem se tornando cada vez mais promissor.

\section{AGRADECIMENTOS}

Os autores agradecem o apoio da FAPESP pelo suporte financeiro. 


\section{REFERÊNCIAS}

1. Alegret, S.; Analyst 1996, 121, 1751.

2. Fleet, B.; Gunasingham, H.; Talanta 1992, 39, 1449.

3. Wring, S. A.; Hart, J. P.; Analyst 1992, 117, 1215.

4. Wang, J.; Electroanalysis 1991, 3, 255.

5. Brett, C. M. A.; Brett, A. M. O. Em Electrochemistry - Principles, Methods and Applications; Oxford University Press: New York, 1994, p. 339.

6. Rieger, P. H. Em Electrochemistry; Chapman \& Hall: New York, 1994, p. 109.

7. Bard, A.; Faulkner, L. R.; Electrochemical Methods - Fundamentals and Applications, John Wiley \& Sons: New York, 1980, p. 370.

8. Hulanicki, A.; Glab, S.; Ingman, F.; Pure Appl. Chem. 1991, 63, 1247.

9. Fatibello-Filho, O.; Capelato, M. D. ; Quim. Nova, 1992, 15, 28.

10. Mulchandani, A.; Bassi, A. S.; Crit. Rev. Biotechnol. 1995, 15, 105.

11. Turner, A. P. F.; Karube, Y.; Wilson, G. S.; Biosensors. Fundamentals and Applications, Oxford University Press: New York, 1987.

12. Zhang, Y.; Chen, N. Y.; Zhu, L. G.; Chem. J. Chin. Univers. 2000, 21, 675.

13. Iwuoha, E. I.; Kane, S.; Ania, C. O.; Smyth, M. R.; De Montellano, P. R. O.; Fuhr, U.; Electroanalysis 2000, 12, 980.

14. Bottcher, H.; J. Prakt. Chemie-Pract. Appl. Chem. 2000, 342, 427.

15. Bhatia, R. B.; Brinker, C. J.; Gupta, A. K.; Singh, A. K.; Chem. Mater. 2000, 12, 2434.

16. Wolfbeis, O. S.; Anal. Chem. 2000, 72, 81R.

17. Badjic, J. D.; Kostic, N. M.; Chem. Mater. 1999, 11, 3671.

18. Niu, J. J.; Lee, J. Y.; Sens. Actuators, B 2000, 62, 190.

19. Wolfbeis, O. S.; Oehme, I.; Papkovskaya, N.; Klimant, I.; Biosens. Bioelectron. 2000, 15, 69.

20. Li, Q. W.; Luo, G. A.; Wang, Y. M.; Zhang, X. R.; Mater. Sci. Eng., C 2000, $11,67$.

21. Cass, T.; Ligler, F. S.; Immobilized Biomolecules in Analysis: A Practical Approach, Oxford Univ. Press: New York, 1998.

22. Cunninggham, A. J. Em Introduction to Bioanalytical Sensors; John Wiley \& Sons, Inc.: New York, 1998, cap. 3.

23. Scouten, W. H.; Luong, J. H. T.; Brown, R. S.; Tibtech. 1998, 13, 178.

24. De La Guardia, M.; Mikrochim. Acta 1995, 120, 243.

25. Arnold, M. A.; Meyerhoff, M. E.; Crit. Rev. Anal. Chem. 1988, 20, 149.

26. Li, J.; Tan, S. N.; Anal. Lett. 2000, 33, 1467.

27. Hiratsuka, R. S.; Santili, C. V.; Pulcinelli, S. H.; Quim. Nova 1995, $18,171$.

28. Brinker, C. J.; Scherer, G. W.; Sol-Gel Science - The Physics and Chemistry of Sol-Gel Processing, Academic Press, Inc.: San Diego, 1990.

29. Brinker, C. J.; J. Non-Cryst. Solids 1988, 100, 31.

30. Hench, L. L.; West, J. K.; Chem. Rev. 1990, 90, 33.

31. Corriu, R. J. P.; Leclercq, D.; Angew. Chem., Int. Ed. 1996, 35, 1420.

32. Buckley, A. M.; Greenblatt, M.; J. Chem. Educ. 1994, 71, 599.

33. Wen, J.; Wilkes, G. L.; Chem. Mater. 1996, 8,1667.

34. Mackenzie, J. D.; Chung, Y. J.; Hu, Y.; J. Non-Cryst. Solids 1992, $147-$ $148,271$.

35. Li, X.; King, T. A.; J. Non-Cryst. Solids 1996, 204, 235.

36. Ou, D. L.; Seddon, A. B.; J. Non-Cryst. Solids 1997, 210, 187.

37. Schmidt, H.; Scholze, H.; Kaiser, A.; J. Non-Cryst. Solids 1994, 63, 1.

38. Schubert, U.; Husing, N.; Lorenz, A.; Chem. Mater. 1995, 7, 2010.

39. Deschler, U.; Kleinschmit, P.; Panster, P.; Angew. Chem., Int. Ed. 1986, $25,236$.

40. Braun, S.; Rappoport, S.; Zusman, R.; Avinir, D.; Ottolenghi, M.; Mater. Lett. 1990, 10, 1.

41. Braun, S.; Rappoport, S.; Zusman, R.; Shteltzer, S.; Drukman, S.; Avnir, D.; Ottolenghi, M. Em Biotechnology: Bridging Research and Applications; Kamely, D.; Chakrabarty, A.; Kornguth, S. E., eds.; Kluver Academic Publishers: Amsterdan, 1991, p. 205.

42. Ellerby, L. M.; Nishida, C. R.; Nishida, F.; Yamanaka, S. A.; Dunn, B.; Valentine, J. S.; Zink, J. I.; Science 1992, 225, 1113.

43. Shtelzer, S.; Rappoport, D.; Avnir, D.; Ottolenghi, M.; Braun, S.; Appl. Biochem. 1992, 15, 227.

44. Reetz, M. T.; Zonta, A.; Simpelkamp, J.; Angew. Chem., Int. Ed. 1995, 34, 301.

45. Avnir, D.; Braun, S.; Lev, O.; Ottolenghi, M.; Chem. Mater. 1994, 6, 1605.

46. Peng, T.; Cheng, Q.; Stevens, R. C.; Anal. Chem. 2000, 72, 1611.

47. Lee, W.-Y.; Lee, K. S.; Kim, T.-H.; Shin, M.-C.; Park, J.-K.; Electroanalysis 2000, 12, 78.

48. Yamada, S.A.; Charych, D. H.; Loy, D. A.; Sasaki, D. Y.; Langmuir 1997, 13,5049

49. Lev, O.; Tsionsky, M.; Rabinovich, L.; Glezer, V.; Sampath, S.; Pankratov, I.; Gun, J.; Anal. Chem. 1995, 67, 22A.
50. Dave, C.; Dunn, B.; Valentine, J. S.; Zink, J.; Anal. Chem. 1994, 66, 1120A.

51. Lev, O.; Wu, Z.; Bharathi, S.; Glezer, V.; Modestov, A.; Gun, J.; Rabinovich, L.; Sampath, S.; Chem. Mater. 1997, 9, 2354.

52. Liu, Z.; Deng, J.; Li, D.; Anal. Chim. Acta 2000, 407, 87.

53. Da Silva, L. R. D.; Gushikem, Y.; Kubota, L. T.; Colloids Surf., B 1996, $6,309$.

54. Coche-Guérente, L.; Cosnier, S.; Labbé, P.; Chem. Mater. 1997, 9, 1348.

55. Wang, J.; Park, D. S.; Pamidi, P. V. A.; J. Electroanal. Chem. 1997, 434, 185.

56. Sampath, S.; Lev, O.; Electroanalysis 1996, 8, 1112.

57. Wang, J.; Pamidi, P. V. A.; Anal. Chem. 1997, 69, 4490.

58. Alber, K. S.; Cox, J. A.; Mikrochim. Acta 1997, 127, 131.

59. Tatsu, Y.; Yamashita, K.; Yamaguchi, M.; Yamamura, S.; Yamamoto, H.; Yoshikawa, S.; Chem. Lett. 1992, 1619.

60. Audebert, P.; Demaille, C.; Sanchez, C.; Chem. Mater. 1993, 5, 911.

61. Narang, U.; Prasad, P. N.; Bright, F. V.; Ramanathan, K.;Kumar, N.D.; Malhotra, B. D.; Kamalasanan, M. N.; Chandra, S.; Anal. Chem. 1994, 66, 3139.

62. Li, J.; Tan, S. N.; Ge, H.; Anal. Chim. Acta 1996, 335, 137.

63. Yang, Q.; Atanasov, P.; Wilkins, E.; Hughes, R. C.; Anal. Lett. 1995, 28, 2439.

64. Park, T. M.; Iwuoha, I.; Smyth, M. R.; MacCraith, B. D.; Anal. Commun. 1996, 33, 271.

65. Glezer, V.; Lev, O.; J. Am. Chem. Soc. 1993, 115, 2533.

66. Tsionsky, M.; Gun, G.; Glezer, V.; Lev, O.; Anal. Chem. 1994, 66, 1747.

67. Pankratov, I.; Lev, O.; J. Electroanal. Chem. 1995, 393, 35.

68. Sampath, S.; Lev, O.; Electroanalysis 1996, 8, 1112.

69. Gun, J.; Lev, O.; Anal. Lett. 1996, 29, 1933.

70. Gun, J.; Lev, O.; Anal. Chim. Acta 1996, 336, 95.

71. Sampath, S.; Lev, O.; Adv. Mater. 1997, 9, 410.

72. Sampath, S.; Lev, O.; Anal. Chem. 1996, 68, 2015.

73. Sampath, S.;Lev, O.; J. Electroanal. Chem. 1997, 426, 13.

74. Wang, J.; Parsad, V. A.; Park, D. S.; Electroanalysis 1997, 9, 52.

75. Wang, J.; Pamidi, V. A.; Park, D. S.; Anal. Chem. 1996, 68, 2705.

76. Gerritsen, M.; Kros, A.; Sprakel, V.; Lutterman, J. A.; Nolte, R. J. M.; Jansen, J. A.; Biomaterials 2000, 21, 71.

77. Sasaki, D. Y.; Loy, D. A.; Yamanaka, S. A.; Immobilized lipid-bilayer materials in a silica matrix US 6048546 A, 2000.

78. Schubert, U.; Dalton 1996, 3343.

79. Hsueh, C.; Collinson, M. M.; J. Electroanal. Chem. 1997, 420, 243.

80. Gill, I.; Ballestros, A.; J. Am. Chem. Soc. 1998, $120,8587$.

81. Raman, N.; Anderson, M.; Brinker, C. J.; Chem. Mater. 1996, 8, 1682.

82. Makote, R.; Collinson, M. M.; Chem. Mater. 1998, 10, 2440.

83. Wang, J.; Pamidi, P. V.; Zanette, D.; J. Am. Chem. Soc. 1998, 120, 5852.

84. Yao, T.; Takashima, K.; Biosens. Bioelectron. 1998, 13, 67.

85. Verghese, M.; Ramanathan, K.; Ashraf, S.; Kamalasanan, M.; Malhorta, B.; Chem. Mater. 1996, 8, 822.

86. Wang, K. M.; Li, J.; Yang, X. H.; Shen, F. L.; Wang, X.; Sens. Actuators, B 2000, 65, 239.

87. Yang, S. P.; Lu, Y. F.; Atanassov, P.; Wilkins, E.; Long, X. C.; Talanta 1998, 47, 735 .

88. Li, J.; Wang, K. M.; Xiao, D.; Yang, X. H.; Chem. J. Chin. Univers.-Chin. 2000, 21, 1018.

89. Park, T. M.; Anal. Lett. 1999, 32, 287.

90. Barker, S. L. R.; Zhao, Y. D.; Marletta, M. A.; Kopelman, R.; Anal. Chem. 1999, 71, 2071.

91. Lee, W. Y.; Kim, S. R.; Kim, T. H.; Lee, K. S.; Shin, M. C.; Park, J. K.; Anal. Chim. Acta 2000, 404, 195.

92. Metzger, J.; Reiss, M.; Hartmeier, W.; Biosens. Bioelectron. 1998, 13, 1077.

93. Peng, T. Z.; Chen, Q.; Stevens, R. C.; Anal. Chem. 2000, 72, 1611.

94. Pandey, P. C.; Upadhyay, S.; Pathak, H. C.; Pandey, C. M. D.; Tiwari, I.; Sens. Actuators, B 2000, 62, 109.

95. Lee, W. Y.; Lee, K. S.; Kim, T. H.; Shin, M. C.; Park, J. K.; Electroanalysis 2000, $12,78$.

96. Singh, A. K.; Flounders, A. W.; Volponi, J. V.; Ashley, C. S.; Wally, K.; Schoeniger, J. S.; Biosens. Bioelectron. 1999, 14, 703.

97. Wang, B.; Zhang, J.; Cheng, G.; Dong, S.; Anal. Chim. Acta 2000, 407, 111.

98. Nguyen, T.; McNamara, K. P.; Rosenzweig, Z.; Anal. Chim. Acta 1999, $400,45$.

99. Wang, B.; Dong, S.; Talanta 2000, 51, 565.

100. Zhang, J.; Li, B.; Wang, Z.; Cheng, G.; Dong, S.; Anal. Chim. Acta 1999, $388,71$.

101. Williams, A. K.; Hupp, J. T.; J. Am. Chem. Soc. 1998, 120, 4366. 